\title{
Chironomidae Types at the Museum of Comparative Zoology, Boston
}

\author{
Fabio Laurindo da Silva \\ Laboratory of Aquatic Entomology, Department of Hydrobiology, Federal University of São Carlos, P.O. \\ Box 676, 13565-905, São Carlos, SP, Brazil. \\ Section of Natural History, Museum of Natural History and Archaeology, Norwegian University of Sci- \\ ence and Technology, NO-7491, Trondheim, Norway. \\ Ernst Mayr Fellow, Museum of Comparative Zoology, Harvard University, Massachusetts, USA. \\ E-mail:fabelha@hotmail.com
}

\begin{abstract}
The Chironomidae (Insecta: Diptera) type collection at the Museum of Comparative Zoology (MCZ) is reviewed. It comprises 23 primary types, as well as paratypes and paralectotypes for an additional 29 species, mostly resulting from research by H. Loew and H. K. Townes, respectively. Notes updating the taxonomic status are provided for several species.
\end{abstract}

\section{Introduction}

The chironomid collection of the Museum of Comparative Zoology (MCZ) at Harvard University in Boston, Massachusetts (USA), includes about 1,200 specimens mostly stored dry on pins, with a small number mounted on slides. It is historically relevant but not well known. The main parts of this material have resulted from respective research endeavors by H. Loew (1807-1879) and H. K. Townes (1913-1990). Its geographic emphasis lies in the United States and Central America.

The annotated checklist presented below (Table 1) was generated during a research visit to the $\mathrm{Mu}-$ seum of Comparative Zoology in February 2011 that was partly financed by an Ernst Mayr Travel Grant in Animal Systematics from MCZ/Harvard University.

\section{Results}

The collection includes 52 type specimens now classified as 10 holotypes, 5 lectotypes and 8 syntypes, as well as paratypes and paralectotypes of an additional 29 Chironomidae species (Table 1). The majority of the types belong to the subfamilies Chironominae and Tanypodinae.

All names of species and references were checked with the corresponding literature. The notes on the species' taxonomic status are based on Systema Dipterorum (Thompson \& Pape 2010), as well as on published papers, catalogs (Oliver et al. 1990, Spies \& Reiss 1996, Ashe \& O'Connor 2009) and/ or information on the collection labels. Some of the primary types were found labeled merely with "type". The present respective interpretations of such specimens as holotypes, syntypes, etc., are based on all available data, e.g. those in the respective corresponding publications.

The information presented here will be included in the database of primary types of Chironomidae already accessible online as part of the MCZ Entomology database (http://insects.oeb.harvard.edu/ $\underline{\mathrm{mcz}})$.

Table 1. Chironomidae species with types deposited in the collection of the Museum of Comparative Zoology at Harvard University, Boston. Abbreviations: $\mathrm{H}=$ holotype, $\mathrm{L}=$ lectotype, $\mathrm{P}=$ paratype, $\mathrm{PL}=$ paralectotype, $\mathrm{S}=$ syntype, $\mathrm{M}=$ male, $\mathrm{F}=$ female, $\mathrm{AU}=$ Australian, $\mathrm{NE}=$ Nearctic, $\mathrm{NT}=$ Neotropical

CHIRONOMINAE

\begin{tabular}{|c|c|c|c|c|c|c|c|c|}
\hline $\begin{array}{c}\text { Type } \\
\text { number }\end{array}$ & Original genus & $\begin{array}{l}\text { Original } \\
\text { species }\end{array}$ & $\begin{array}{l}\text { Author/ } \\
\text { Reference }\end{array}$ & Type & Sex & Preparation & Distribution & Note \\
\hline $\begin{array}{l}\text { MCZT } \\
07433\end{array}$ & Chironomus & brachialis & $\begin{array}{l}\text { Coquillett, } \\
\text { 1901: } 607\end{array}$ & S & $\mathrm{M}, \mathrm{F}$ & Pinned & $\mathrm{NE}$ & 1 \\
\hline $\begin{array}{l}\text { MCZT } \\
19425\end{array}$ & Chironomus & bulbosa & $\begin{array}{c}\text { Gerry, 1933: } \\
97\end{array}$ & S & $\mathrm{M}, \mathrm{F}$ & Pinned & NT & 2 \\
\hline $\begin{array}{l}\text { MCZT } \\
15655\end{array}$ & Chironomus & imperator & $\begin{array}{l}\text { Walley, 1926: } \\
64\end{array}$ & $\mathrm{P}$ & $\mathrm{M}, \mathrm{F}$ & Pinned & $\mathrm{NE}$ & 3 \\
\hline
\end{tabular}




\begin{tabular}{|c|c|c|c|c|c|c|c|c|}
\hline $\begin{array}{l}\text { MCZT } \\
19424\end{array}$ & Chironomus & jamaicensis & $\begin{array}{c}\text { Gerry, 1933: } \\
96\end{array}$ & $\mathrm{~S}$ & $\mathrm{M}, \mathrm{F}$ & Pinned & NT & 4 \\
\hline $\begin{array}{l}\text { MCZT } \\
25385\end{array}$ & Chironomus & nigricans & $\begin{array}{l}\text { Johannsen, } \\
\text { 1905: } 219\end{array}$ & $\mathrm{P}$ & $\mathrm{M}, \mathrm{F}$ & Pinned & NE & 5 \\
\hline $\begin{array}{l}\text { MCZT } \\
07434\end{array}$ & Chironomus & taeniapennis & $\begin{array}{l}\text { Coquillett, } \\
\text { 1901: } 607\end{array}$ & $\mathrm{~S}$ & $\mathrm{~F}$ & Pinned & $\mathrm{NE}$ & 6 \\
\hline $\begin{array}{l}\text { MCZT } \\
25404\end{array}$ & Glyptotendipes & testaceus & $\begin{array}{c}\text { Townes, 1945: } \\
140\end{array}$ & $\mathrm{P}$ & $\mathrm{M}, \mathrm{F}$ & Pinned & NE & \\
\hline $\begin{array}{l}\text { MCZT } \\
25393\end{array}$ & Harnischia & amachaerus & $\begin{array}{c}\text { Townes, 1945: } \\
168\end{array}$ & $\mathrm{P}$ & M & Pinned & NE & 7 \\
\hline $\begin{array}{l}\text { MCZT } \\
25381\end{array}$ & Harnischia & argentea & $\begin{array}{c}\text { Townes, 1945: } \\
164\end{array}$ & $\mathrm{P}$ & M & Pinned & $\mathrm{NE}$ & 8 \\
\hline $\begin{array}{l}\text { MCZT } \\
25389\end{array}$ & Harnischia & carinata & $\begin{array}{c}\text { Townes, 1945: } \\
158\end{array}$ & $P$ & M & Pinned & $\mathrm{NE}$ & 9 \\
\hline $\begin{array}{l}\text { MCZT } \\
25398\end{array}$ & Harnischia & cuneata & $\begin{array}{c}\text { Townes, 1945: } \\
163\end{array}$ & $\mathrm{P}$ & M & Pinned & NE & 10 \\
\hline $\begin{array}{l}\text { MCZT } \\
25401\end{array}$ & Harnischia & potamogeti & $\begin{array}{c}\text { Townes, 1945: } \\
159\end{array}$ & $P$ & M & Pinned & $\mathrm{NE}$ & 11 \\
\hline $\begin{array}{l}\text { MCZT } \\
25387\end{array}$ & Omisus & pica & $\begin{array}{c}\text { Townes, 1945: } \\
27\end{array}$ & $\mathrm{P}$ & M & Pinned & NE & \\
\hline $\begin{array}{l}\text { MCZT } \\
25394\end{array}$ & Polypedilum & simulans & $\begin{array}{c}\text { Townes, 1945: } \\
43\end{array}$ & $\mathrm{P}$ & M & Pinned & NE & \\
\hline $\begin{array}{l}\text { MCZT } \\
25399\end{array}$ & Pseudochironomus & banksi & $\begin{array}{c}\text { Townes, 1945: } \\
17\end{array}$ & $\mathrm{H}, \mathrm{P}$ & M & Pinned & $\mathrm{NE}$ & \\
\hline $\begin{array}{l}\text { MCZT } \\
25386\end{array}$ & Pseudochironomus & crassus & $\begin{array}{c}\text { Townes, 1945: } \\
15\end{array}$ & $\mathrm{H}$ & M & Pinned & NE & \\
\hline $\mathrm{MCZT}$ & Stenochironomus & albipalpus & $\begin{array}{l}\text { Borkent, } \\
\text { 1984: } 66\end{array}$ & $\mathrm{P}$ & $\mathrm{M}, \mathrm{F}$ & Slide & $\mathrm{NE}$ & \\
\hline $\mathrm{MCZT}$ & Stenochironomus & fuscipatellus & $\begin{array}{l}\text { Borkent, } \\
\text { 1984: } 64\end{array}$ & $P$ & M & Slide & NE & \\
\hline MCZT & Stenochironomus & woodi & $\begin{array}{l}\text { Borkent, } \\
\text { 1984: } 91\end{array}$ & $\mathrm{P}$ & $\mathrm{M}, \mathrm{F}$ & Slide & NE & \\
\hline $\begin{array}{l}\text { MCZT } \\
27258\end{array}$ & Tanytarsus & subtendens & $\begin{array}{c}\text { Townes, 1945: } \\
65\end{array}$ & $\mathrm{P}$ & M, F & Pinned & $\mathrm{NE}$ & 12 \\
\hline $\begin{array}{l}\text { MCZT } \\
25391\end{array}$ & Tribelos & protextus & $\begin{array}{c}\text { Townes, 1945: } \\
69\end{array}$ & $\mathrm{P}$ & M & Pinned & NE & 13 \\
\hline $\begin{array}{l}\text { MCZT } \\
25403\end{array}$ & Tendipes & atroviridis & $\begin{array}{c}\text { Townes, 1945: } \\
114\end{array}$ & $\mathrm{P}$ & M. F & Pinned & $\mathrm{NE}$ & 14 \\
\hline $\begin{array}{l}\text { MCZT } \\
25384\end{array}$ & Tendipes & biseta & $\begin{array}{c}\text { Townes, 1945: } \\
127\end{array}$ & $\mathrm{P}$ & $\mathrm{M}, \mathrm{F}$ & Pinned & $\mathrm{NE}$ & 15 \\
\hline $\begin{array}{l}\text { MCZT } \\
25395\end{array}$ & Tendipes & carus & $\begin{array}{c}\text { Townes, 1945: } \\
118\end{array}$ & $\mathrm{P}$ & M & Pinned & $\mathrm{NE} / \mathrm{NT}$ & 16 \\
\hline $\begin{array}{l}\text { MCZT } \\
25397\end{array}$ & Tendipes & ochreatus & $\begin{array}{c}\text { Townes, 1945: } \\
115\end{array}$ & $\mathrm{P}$ & M & Pinned & $\mathrm{NE}$ & 17 \\
\hline $\begin{array}{l}\text { MCZT } \\
25382\end{array}$ & Tendipes & tuberculatus & $\begin{array}{c}\text { Townes, 1945: } \\
128\end{array}$ & $\mathrm{H}, \mathrm{P}$ & $\mathrm{M}, \mathrm{F}$ & Pinned & $\mathrm{NE}$ & 18 \\
\hline
\end{tabular}

ORTHOCLADIINAE

\begin{tabular}{|c|c|c|c|c|c|c|c|c|}
\hline $\begin{array}{c}\text { Type } \\
\text { number }\end{array}$ & Original genus & $\begin{array}{c}\text { Original } \\
\text { species }\end{array}$ & $\begin{array}{c}\text { Author/ } \\
\text { Reference }\end{array}$ & Type & Sex & Preparation & Distribution & Note \\
\hline $\begin{array}{l}\text { MCZT } \\
01260\end{array}$ & Chasmatonotus & bimaculatus & $\begin{array}{c}\text { Osten-Sacken, } \\
\text { 1877: } 191\end{array}$ & $\mathrm{~S}$ & M & Pinned & $\mathrm{NE}$ & \\
\hline $\begin{array}{c}\text { MCZT } \\
10388\end{array}$ & Chasmatonotus & unimaculatus & $\begin{array}{c}\text { Loew, 1864: } \\
50\end{array}$ & $\mathrm{~S}$ & $\mathrm{M}, \mathrm{F}$ & Pinned & $\mathrm{NE}$ & \\
\hline
\end{tabular}


TANYPODINAE

\begin{tabular}{|c|c|c|c|c|c|c|c|c|}
\hline $\begin{array}{c}\text { Type } \\
\text { number }\end{array}$ & Original genus & $\begin{array}{c}\text { Original } \\
\text { species }\end{array}$ & $\begin{array}{c}\text { Author/ } \\
\text { Reference }\end{array}$ & Type & Sex & Preparation & Distribution & Note \\
\hline MCZT & Ablabesmyia & parajanta & $\begin{array}{c}\text { Roback, 1971: } \\
373\end{array}$ & $\mathrm{P}$ & M & Slide & NE & \\
\hline MCZT & Ablabesmyia & tarella & $\begin{array}{c}\text { Roback, 1971: } \\
368\end{array}$ & $\mathrm{P}$ & M & Pinned/Slide & NE & 19 \\
\hline $\begin{array}{l}\mathrm{MCZT} \\
31778\end{array}$ & Coelotanypus & cletis & $\begin{array}{c}\text { Roback, 1963: } \\
174\end{array}$ & $\mathrm{H}$ & M & Slide & NT & \\
\hline $\begin{array}{l}\text { MCZT } \\
10367\end{array}$ & Tanypus & bellus & Loew, 1866: 4 & $\mathrm{~L}, \mathrm{PL}$ & $\mathrm{M}, \mathrm{F}$ & Pinned/Slide & NE & 20 \\
\hline $\begin{array}{l}\text { MCZT } \\
19423\end{array}$ & Tanypus & brooksi & $\begin{array}{l}\text { Gerry, 1933: } \\
95\end{array}$ & S & M & Pinned & NT & 21 \\
\hline $\begin{array}{c}\text { MCZT } \\
07431\end{array}$ & Tanypus & concinnus & $\begin{array}{l}\text { Coquillett, } \\
\text { 1895: } 308\end{array}$ & S & $\mathrm{M}, \mathrm{F}$ & Pinned & $\mathrm{NE} / \mathrm{NT}$ & 22 \\
\hline $\begin{array}{c}\text { MCZT } \\
15660\end{array}$ & Tanypus & cornuticaudatus & $\begin{array}{c}\text { Walley, 1925: } \\
277\end{array}$ & $\mathrm{P}$ & $\mathrm{M}, \mathrm{F}$ & Pinned/Slide & NE & 23 \\
\hline $\begin{array}{l}\text { MCZT } \\
15657\end{array}$ & Tanypus & currani & $\begin{array}{c}\text { Walley, 1925: } \\
276\end{array}$ & $\mathrm{P}$ & $\mathrm{F}$ & Pinned & NE & 24 \\
\hline $\begin{array}{c}\text { MCZT } \\
10368\end{array}$ & Tanypus & flavicinctus & $\begin{array}{c}\text { Loew, 1861: } \\
309\end{array}$ & $\mathrm{H}$ & M & Slide & NE & 25 \\
\hline $\begin{array}{c}\mathrm{MCZT} \\
15658\end{array}$ & Tanypus & garretti & $\begin{array}{c}\text { Walley, 1925: } \\
275\end{array}$ & $\mathrm{P}$ & $\mathrm{F}$ & Pinned/Slide & $\mathrm{NE}$ & 26 \\
\hline $\begin{array}{c}\text { MCZT } \\
10369\end{array}$ & Tanypus & hirtipennis & Loew, 1866: 5 & $\mathrm{H}$ & M & Slide & NE & 27 \\
\hline $\begin{array}{l}\text { MCZT } \\
10376\end{array}$ & Tanypus & humeralis & Loew, 1866: 3 & $\mathrm{~L}, \mathrm{PL}$ & $\mathrm{M}, \mathrm{F}$ & Pinned/Slide & NE/NT & 28 \\
\hline $\begin{array}{l}\text { MCZT } \\
15661\end{array}$ & Tanypus & mallochi & $\begin{array}{c}\text { Walley, 1925: } \\
273\end{array}$ & $\mathrm{P}$ & M & Pinned & NE & 29 \\
\hline $\begin{array}{l}\text { MCZT } \\
15662\end{array}$ & Tanypus & peleensis & $\begin{array}{l}\text { Walley, 1926: } \\
\qquad 64\end{array}$ & $\mathrm{P}$ & $\mathrm{M}, \mathrm{F}$ & Pinned/Slide & NE/NT & 30 \\
\hline $\begin{array}{l}\text { MCZT } \\
15656\end{array}$ & Tanypus & pilicaudatus & $\begin{array}{c}\text { Walley, 1925: } \\
277\end{array}$ & $\mathrm{P}$ & M & Pinned/Slide & NE & 31 \\
\hline $\begin{array}{l}\text { MCZT } \\
10370\end{array}$ & Tanypus & pilosellus & Loew, 1866: 5 & $\mathrm{~L}, \mathrm{PL}$ & $\mathrm{F}$ & Pinned/Slide & $\mathrm{NE} / \mathrm{NT}$ & 32 \\
\hline $\begin{array}{c}\text { MCZT } \\
10371\end{array}$ & Tanypus & pinguis & $\begin{array}{c}\text { Loew, 1861: } \\
308\end{array}$ & $\mathrm{H}$ & $\mathrm{F}$ & Pinned & NE & 33 \\
\hline $\begin{array}{l}\text { MCZT } \\
15659\end{array}$ & Tanypus & prudens & $\begin{array}{c}\text { Walley, 1925: } \\
275\end{array}$ & $\mathrm{P}$ & M & Slide & $\mathrm{NE}$ & 34 \\
\hline $\begin{array}{l}\text { MCZT } \\
10372\end{array}$ & Tanypus & pusillus & Loew, 1866: 5 & $\mathrm{H}$ & $\mathrm{M}, \mathrm{F}$ & Pinned & $\mathrm{NE}$ & 35 \\
\hline $\begin{array}{l}\text { MCZT } \\
10373\end{array}$ & Tanypus & scapularis & Loew, 1866: 2 & L, PL & $\mathrm{M}, \mathrm{F}$ & Pinned/Slide & $\mathrm{NE} / \mathrm{NT}$ & 36 \\
\hline $\begin{array}{l}\text { MCZT } \\
31777\end{array}$ & Tanypus & telus & $\begin{array}{c}\text { Roback, 1971: } \\
61\end{array}$ & $\mathrm{H}, \mathrm{P}$ & M. F & Pinned/Slide & $\mathrm{NE}$ & \\
\hline $\begin{array}{l}\text { MCZT } \\
10374\end{array}$ & Tanypus & thoracicus & Loew, 1866: 4 & $\mathrm{~L}, \mathrm{PL}$ & $\mathrm{M}, \mathrm{F}$ & Pinned/Slide & $\mathrm{NE}$ & 37 \\
\hline $\begin{array}{l}\text { MCZT } \\
10375 \\
\end{array}$ & Tanypus & tricolor & $\begin{array}{c}\text { Loew, 1861: } \\
309\end{array}$ & $\mathrm{H}$ & $\mathrm{F}$ & Pinned & $\mathrm{NE} / \mathrm{NT}$ & 38 \\
\hline
\end{tabular}

TELMATOGETONINAE

\begin{tabular}{ccccccccc}
\hline $\begin{array}{c}\text { Type } \\
\text { number }\end{array}$ & Original genus & $\begin{array}{c}\text { Original } \\
\text { species }\end{array}$ & $\begin{array}{c}\text { Author/ } \\
\text { Reference }\end{array}$ & Type & Sex & Preparation & Distribution & Note \\
\hline $\begin{array}{c}\text { MCZT } \\
27639\end{array}$ & Thalassomyia & setosipennis & $\begin{array}{c}\text { Wirth, 1947: } \\
121\end{array}$ & P & M, F & Pinned & AU & 39 \\
\hline
\end{tabular}


Notes on Table 1

Chironomus brachialis Coquillett, 1901 is now Demeijerea brachialis (Coquillett, 1901).

Chironomus bulbosa Gerry, 1933, the spelling of which has been corrected to C. bulbosus, is a nomen dubium in Chironominae.

Chironomus imperator Walley, 1926 is a junior synonym of Chironomus plumosus (Linnaeus, 1758).

Chironomus jamaicensis Gerry, 1933 is a nomen dubium in Chironominae.

Chironomus nigricans Johannsen, 1905 is now Endochironomus nigricans (Johannsen, 1905).

Chironomus taeniapennis Coquillett, 1901 is a junior synonym of Stenochironomus hilaris (Walker, 1848).

Harnischia amachaerus Townes, 1945 is now Cladopelma amachaerus (Townes, 1945).

Harnischia argentea Townes, 1945 is now Cyphomella argentea (Townes, 1945).

Harnischia carinata Townes, 1945 is now Parachironomus carinatus (Townes, 1945).

Harnischia cuneata Townes, 1945 is now Demicryptochironomus cuneatus (Townes, 1945).

Harnischia potamogeti Townes, 1945 is now Parachironomus potamogeti (Townes, 1945).

Tanytarsus subtendens Townes, 1945 is now Endochironomus subtendens (Townes, 1945).

Tanytarsus protextus Townes, 1945 is now a junior synonym of Tribelos jucundum (Walker, 1848).

Tendipes atroviridis Townes, 1945 is now Chironomus atroviridis (Townes, 1945).

Tendipes biseta Townes, 1945 is now Chironomus biseta (Townes, 1945).

Tendipes carus Townes, 1945 is now Goeldichironomus carus (Townes, 1945).

Tendipes ochreatus Townes, 1945 is now Chironomus ochreatus (Townes, 1945).

Tendipes tuberculatus Townes, 1945 is now Chironomus tuberculatus (Townes, 1945).

Ablabesmyia tarella Roback, 1971 is a junior synonym of Ablabesmyia mallochi (Walley, 1925).

Tanypus bellus Loew, 1866 is now Procladius bellus (Loew, 1866).

Tanypus brooksi Gerry, 1933 is nomen dubium in Pentaneurini.

Tanypus concinnus Coquillett, 1895 is now Coelotanypus concinnus (Coquillett, 1895).

Tanypus cornuticaudatus Walley, 1925 is now Helopelopia cornuticaudata (Walley, 1925).

Tanypus currani Walley, 1925 is now Conchapelopia currani (Walley, 1925).

Tanypus flavicinctus Loew, 1861 is a junior synonym of Clinotanypus pinguis (Loew, 1861).

Tanypus garretti Walley, 1925 is a junior synonym of Psectrotanypus dyari (Coquillett, 1902).

Tanypus hirtipennis Loew, 1866 is a junior synonym of Macropelopia decedens (Walker, 1848).

Tanypus humeralis Loew, 1866 is now Coelotanypus humeralis (Loew, 1866).

Tanypus mallochi Walley, 1925 is now Ablabesmyia mallochi (Walley, 1925).

Tanypus peleensis Walley, 1926 is now Ablabesmyia peleensis (Walley, 1926).

Tanypus pilicaudatus Walley, 1925 is now Helopelopia pilicaudata (Walley, 1925).

Tanypus pilosellus Loew, 1866 is now Labrundinia pilosella (Loew, 1866).

Tanypus pinguis Loew, 1861 is now Clinotanypus pinguis (Loew, 1861).

Tanypus prudens Walley, 1925 is a junior synonym of Ablabesmyia pulchripennis (Lundbeck, 1898).

Tanypus pusillus Loew, 1866 is a junior synonym of Procladius bellus (Loew, 1866).

Tanypus scapularis Loew, 1866 is now Coelotanypus scapularis (Loew, 1866).

Tanypus thoracicus Loew, 1866 is a junior synonym of Clinotanypus pinguis (Loew, 1861).

Tanypus tricolor Loew, 1861 is now Coelotanypus tricolor (Loew, 1861).

Thalassomyia setosipennis Wirth, 1947 is valid with the spelling Thalassomya setosipennis. 


\section{Acknowledgements}

I am very grateful to Dr. Philip Perkins, Museum of Comparative Zoology, for his hospitality during my stay, and to Dr. James Hanken and Catherine Weisel for helping me organize my visit. Thanks also to Martin Spies, Dr. Marion Kotrba, Dr. Susana Trivinho-Strixino and Dr. Dalva Matos for supporting my application for an Ernst Mayr Travel Grant, and to Dr. Alaide Fonseca-Gessner for valuable suggestions on the manuscript. Finally, I would like to thank Zaki Habib-Gómez for checking the English text. The author has received financial support from the Ernst Mayr Fund (MCZ - Harvard University) and the National Council for Scientific and Technological Development (CNPq - Brazil).

\section{References}

Ashe P. \& O’Connor J. P. 2009. A world catalogue of Chironomidae (Diptera). Part 1. Buchonomyiinae, Chilenomyiinae, Podonominae, Aphroteniinae, Tanypodinae, Usambaromyiinae, Diamesinae, Prodiamesinae and Telmatogetoninae. Ireland: The National Museum of Ireland, $455 \mathrm{pp}$.

Borkent, A. 1984. The systematics and phylogeny of the Stenochironomus complex (Xestochironomus, Harrisius, and Stenochironomus) (Diptera: Chironomidae). - Mem. Entomol. Soc. Can. 128: 1-269.

Coquillett, D. W. 1895. Descriptions of new genera and species, pp. 307-319. In C. W. Johnson, Diptera of Florida. - Proc. Acad. Nat. Sci. Philadelphia 47: 303-340.

Coquillett, D. W. 1901. New Diptera in the U. S. National Museum. - Proc. U. S. Natl. Mus. 23: 593-618.

Gerry, B. I. 1933. Four new species of Chironomidae from the Greater Antilles. - Psyche, 40: 94-97.

Johannsen, O. A. 1905. Aquatic nematocerous Diptera II, pp. 76-315. In: Needham, J. G., Morton, K. J. and Johannsen, O. A. May flies and midges of New York. Third report on aquatic insects. - Bull. N. Y. State Mus. Sci. Serv. 86: 7-352.
Loew, H. 1861. Diptera Americae septentrionalis indigena. Centuria prima. - Berl. Entomol. Z.

5: 307-359.

Loew, H. 1864. Diptera Americae septentrionalis indigena. Centuria quinta. - Berl. Entomol. Z. 8: 49-104.

Loew, H. 1866. Diptera Americae septentrionalis indigena. Centuria septima. - Berl. Entomol. Z. 10: $1-54$.

Oliver, D. R., Dillon, M. E. \& Cranston, P. S. 1990. A catalog of Nearctic Chironomidae. - Agric. Can. Publ. 1857/B: 1-89.

Osten Sacken, C. R. 1877. Western Diptera: description of new genera and species of Diptera from the region west of the Mississippi and especially from California. - Bull. U. S. Geol. Geogr. Surv. Terr. 3: 189-354.

Roback, S. S. 1963. New Neotropical Coelotanypus (Diptera, Tendipedidae, Pelopiinae). - Entomol. news, 74: 169-176.

Roback, S. S. 1971. The subfamily Tanypodinae in North America. - Monog. Acad. Nat. Sci. Phila., 17: 1-410.

Spies, M. \& F. Reiss. 1996. Catalog and bibliography of Neotropical and Mexican Chironomidae (Insecta, Diptera). - Spixiana Suppl. 22: 61-119.

Thompson, F. C. and T. Pape. 2010. Systema Dipterorum, Version 1.0. http://www.diptera. org/, accessed Feb. 2011.

Townes, H. K. 1945. The Nearctic species of Tendipedini [Diptera, Tendipedidae (= Chironomidae)]. - Am. Midl. Nat. 34: 1-206.

Walley, G. S. 1925. New Canadian Chironomidae of the genus Tanypus (Dip.) - Can. entomol. 57: 271-278.

Walley, G. S. 1926. New Canadian Chironomidae. - Can. entomol. 58: 64-65.

Wirth, W. W. 1947. Notes on the genus Thalassomyia Schiner, with description of two new species (Diptera: Tendipedidae). - Proc. Hawaii Entomol. Soc. 13: 117-139. 